\title{
Cells, Molecules and Mechanisms Involved in the Neuro-Immune Interaction
}

\author{
Rodrigo Pacheco, Francisco Contreras and Carolina Prado
}

Additional information is available at the end of the chapter

http://dx.doi.org/10.5772/48367

\section{Introduction}

The existence of a surveillance and protection system against pathogens and malignant cells has evolved until reach, in the most complex organisms, a very sophisticated immune system composed of an innate arm and an adaptive arm. The innate immune system constitutes the first defense barrier against foreign organisms but it comprises relatively poor diversity of antigen (Ag)-specific recognition elements. In contrast, the adaptive immune system shows a delayed response, and involves numerous Ag-specific recognition elements and highly efficient mechanisms both to eliminate foreign pathogens and tumor cells, and to maintain tolerance to self constituents. $T$ cells are the central players in the adaptive immune response. These cells direct and regulate the function of several immune system cells, thus orchestrating efficient elimination of threats and, at the same time, promoting tolerance to healthy self tissues. Growing evidence has shown that some cells from the adaptive immune system, specially the $\mathrm{T}$ cell compartment, constitute an important link between the nervous system and the immune system. Due to their pivotal role directing immune responses and tolerance, $\mathrm{T}$ cells become strategic target cells to be regulated by the nervous system. On the other hand, evidences point toward T cells not only play an important role driving the elimination of pathogens and tumors and maintaining tolerance to self constituents, but also regulate some nervous system functions such as acquisition of memory and behaviour. This chapter is geared toward analyze and discuss the current knowledge and growing evidences about the interactions between the nervous system and the immune system of the complex organisms. Because mammals present the most complex and sophisticated immune system among the organisms, and most evidences described in the literature correspond to studies of neuro-immune interactions analyzed in these animals, the discussion in this chapter is bounded to mechanisms operating in mammals. The bidirectional interaction between the nervous system and immune system will be analyzed at the level of cellular and molecular mechanisms. In the first half of this 
chapter, interactions between immune and nervous systems will be analyzed in both directions: the immune system mediated regulation of nervous system and the nervous system mediated regulation of immunity. Both kinds of interactions will be analyzed separately for simplification and better understanding. The second half of this chapter will be geared to analyze how immune cells can communicate between them using neurotransmitters and how nervous system cells can also interact with each other using cytokines. Despite this second part of the chapter does not analyze direct neuro-immune interactions, it contributes to understand how both, nervous system and immune system use the same mediators and receptors, which therefore may participate in regulation of immunity, nervous system regulation and also in neuro-immune interactions.

\section{The neuro-immune interaction}

Neuroimmunology studies the interaction between the nervous and the immune system, a relation that can be analyzed in two directions: the nervous system-mediated regulation of immunity and the immune system-mediated regulation of nervous system function. Both kinds of interactions are mediated by complex mechanisms in which participate the same groups of cells and the same kind of molecules (figure 1). These two kinds of neuro-immune interactions will be separately analyzed in this section.

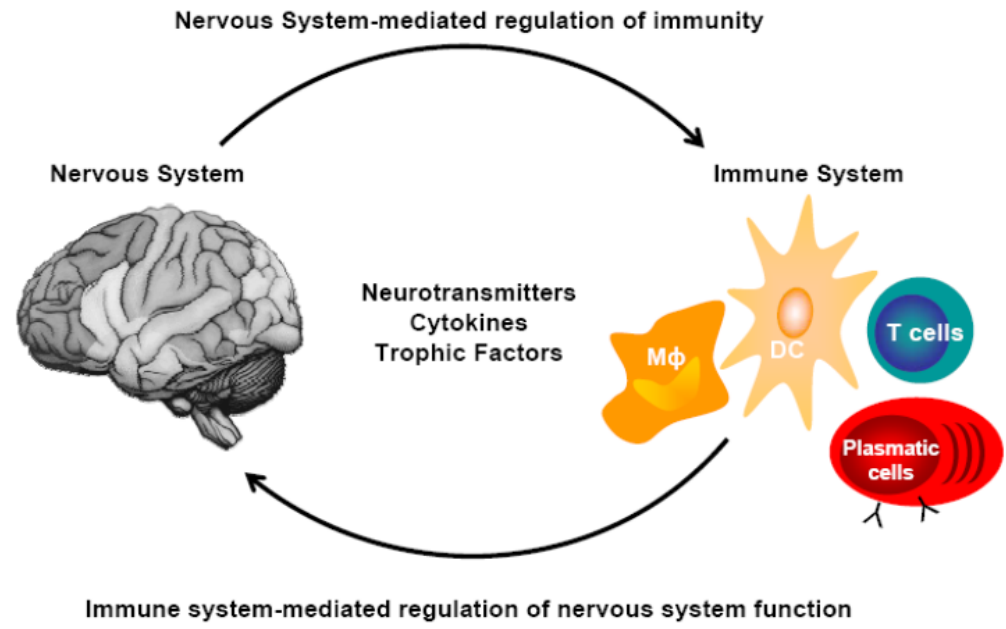

Figure 1. The Neuroimmune interactions. An extensive bidirectional communication takes place between nervous and immune system in both health and disease. The same molecules, including cytokines, neurotransmitters and trophic factors, participate as mediators in both directions.

The first part of this section (section 2.1) will start with a brief introduction about the adaptive immune response. The central role of $\mathrm{T}$ cells in orchestrating the development of immune responses will be highlighted. The development and the role of distinct phenotypic effector $\mathrm{T}$ cells and regulatory $\mathrm{T}$ cells generated during immune response will be summarized. Afterward, in the second part of this section (section 2.2) neurotransmitter 
receptors expressed on immune cells will be described. Circuits by which peripheral nervous system can modulate the function of immune cells will be discussed.

Several studies have shown that immune cells, and mainly $\mathrm{CD}^{+} \mathrm{T}$ cell, contribute significantly to the development and regulation of nervous system processes, such as learning and acquisition of memory. These and other immune cell-mediated mechanisms regulating behaviour in healthy animals will be discussed in the third part of this section (section 2.3). In addition, several studies have shown a key role of T cell-mediated responses in the development and progression of neurodegenerative diseases and others pathologic scenarios occurring in the central nervous system. Examples of these mechanisms occurring during Parkinson's disease, multiple sclerosis and other pathologies will be also discussed in the third part of this section.

\subsection{The central role of $T$ cells and dendritic cells during adaptive immune response}

The immune system has evolved to recognize and eliminate a large variety of Ags that could be potentially harmful for the host. Ag recognition is a highly specific and tightly regulated process, that promotes the destruction of dangerous agents without causing a significant damage to self-constituents. Such a sensitivity and specificity for Ag recognition is mediated by the adaptive arm of the immune system, which is constituted by B and T cells. These cells express Ag-specific receptors on their surfaces that bind to Ags in distinct forms and play very different roles during adaptive immune responses. B cells recognize Ags as soluble molecules and have the ability to differentiate and secrete a soluble form of the B cell receptor called antibody, upon appropriate Ag-stimulation. In contrast, $\mathrm{T}$ cells do not recognize soluble Ags, but require a specialized molecular system for Ag-stimulation. Such a system involves intracellular proteolytic processing of protein Ags into small peptides by Ag-presenting-cells (APCs) and the subsequent loading of these peptides on major histocompatibility complex (MHC) molecules, which are expressed on the surface of APCs. Antigenic peptide-MHC molecule complexes (pMHC) expressed on the APC surface are the ligands for the Ag-receptor of $\mathrm{T}$ cells (TCR). In addition to specific pMHC recognition, $\mathrm{T}$ cell activation can be positively or negatively regulated by a number of nonAg-specific intercellular interactions that are mediated by surface-bound and soluble molecules, which bind to their respective receptors on the $\mathrm{T}$ cell surface. In response to the simultaneous presentation of pathogen or tumor-derived Ags on MHC molecules and costimulatory molecules on the APC surface, Ag-specific naïve T cells are activated. As a result, depending on the nature of the stimuli provided by the APC, T cells undergo massive expansion and differentiate into effector $\mathrm{T}$ cells, which can acquire several effector mechanisms to promote the elimination of pathogens (Wong \& Pamer, 2003) or tumors (Amedei et al., 2011; Park et al., 2011).

Adaptive immune response against foreign proteins requires the activation of Ag-specific $\mathrm{T}$ cells. Help from $\mathrm{T}$ cells contributes to $\mathrm{B}$ cell activation and differentiation into antibody secreting plasma cells. $\mathrm{T}$ cell help is mediated by both soluble and membrane-bound molecules 
(Parker, 1993). In addition, T cell-derived cytokines can also promote or potentiate effector functions of other immune cells, such as macrophages, dendritic cells (DCs), and natural killer (NK) cells. Thus, T cells play a central role coordinating adaptive immune responses (figure 2).

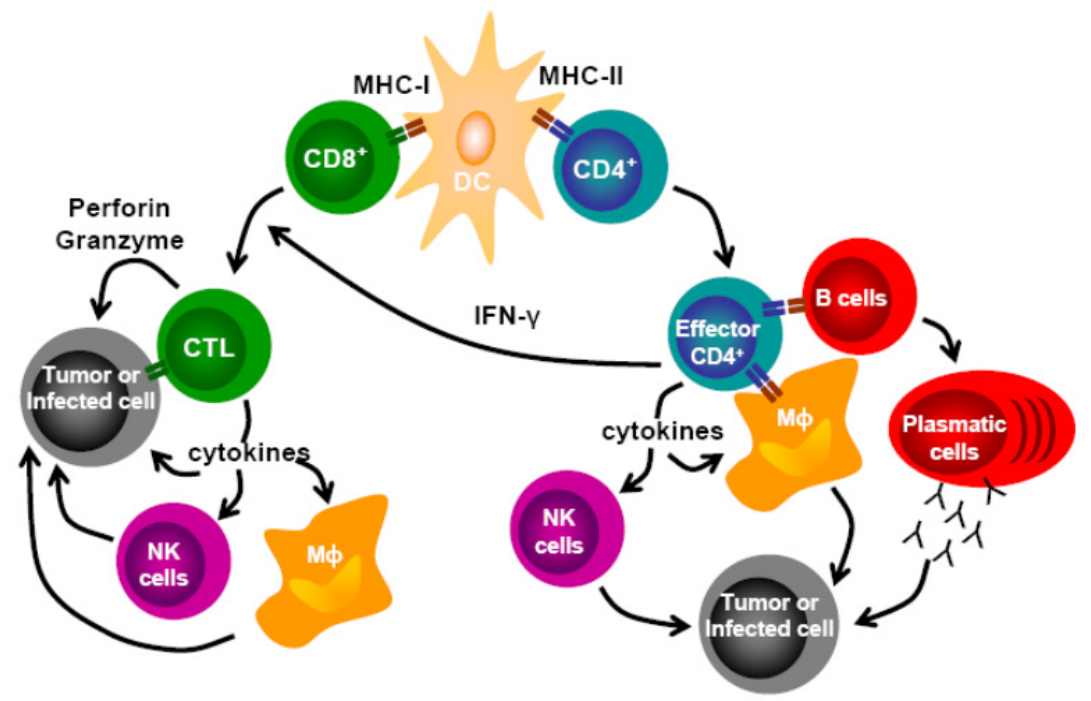

Figure 2. The central role of $\mathrm{T}$ cells and DCs in the adaptive immune response. DCs promote the activation and differentiation of naïve $\mathrm{CD}^{+} \mathrm{T}$ cells to Cytotoxic $\mathrm{T}$ Lymphocytes (CTL) that recognize and kill tumor or infected cells by secreting cytotoxic granules containing perforin and granzymes. In addition, by secreting IFN- $\gamma$, cytotoxic $\mathrm{CD}^{+} \mathrm{T}$ cells may also potentiate function of macrophages and NK cells. On the other hand, DCs activate naïve $\mathrm{CD} 4^{+} \mathrm{T}$ cells and promote their differentiation toward effector $\mathrm{CD} 4^{+} \mathrm{T}$ cells, which contribute to an efficient activation of $\mathrm{CD} 8^{+} \mathrm{T}$ cells and $\mathrm{B}$ cells, and also regulate function of innate immune cells as macrophages and NK cells.

Two main $\mathrm{T}$ cell subsets have been described, which may be phenotypically differentiated by expression on the cell surface of CD4 or CD8, which work as co-receptors for MHC molecules. $\mathrm{CD}^{+}$and $\mathrm{CD}^{+} \mathrm{T}$ cells recognize Ags as peptides bound to class I and class II MHC molecules, respectively. Effector $\mathrm{CD}^{+} \mathrm{T}$ cells may directly recognize tumor or infected cells expressing foreign Ags as surface pMHC complexes and subsequently mediate killing of those cells by secreting cytotoxic granules containing toxic proteins, such as perforin and granzyme (Schoenborn \& Wilson, 2007). In addition, by secreting IFN- $\gamma$, cytotoxic CD8 ${ }^{+} \mathrm{T}$ cells may also potentiate function of other immune cells, including macrophages and NK cells. Hence, CD8 ${ }^{+}$ $\mathrm{T}$ cells are key players during adaptive immune response against intracellular pathogens and tumors (figure 2).

On the other hand, effector $\mathrm{CD} 4{ }^{+} \mathrm{T}$ cells not only contribute to efficient activation of $\mathrm{CD}^{+} \mathrm{T}$ cells (Bennett et al., 1998; Ridge et al., 1998; Schoenberger et al., 1998) and B cells (Smith et al., 2000), but they also regulate the function of several cells of the innate immune system, such as macrophages and NK cells (figure 2). Depending on the signals that DCs provide to T cells in addition to antigenic $\mathrm{pMHC}$, they can promote the differentiation of distinct $\mathrm{CD} 4^{+} \mathrm{T}$ cell 
subsets, including T helper 1 (Th1), Th2 and Th17 (figure 3). A Th1 phenotype on CD4+ T cells is favored by the secretion of IL-12 by DCs. In contrast, a Th2 phenotype is favored in the absence of IL-12 and in the presence of IL-4 during Ag presentation (Watford et al., 2003). Th1 cells secrete predominantly IFN- $\gamma$ and promote protective cellular immunity against intracellular pathogens and tumor cells (figure 3). In addition, IFN- $\gamma$ favors secretion of IgG2a and IgG3 by Ag-specific B cells, both isotypes that efficiently couple Ag recognition with complement activation. In contrast, Th2 cells secrete mainly IL-4, which promote secretion of IgE by B cells, an isotype that couples Ag recognition with mast cell degranulation. In addition, Th2 cells contribute to the activation of eosinophils, promoting allergic-type immune responses that can be efficient at eliminating extracellular pathogens, such as helminths and extracellular bacterium (Del Prete, 1998) (figure 3). Acquisition of Th17 phenotype is promoted by IL-23, TGF- $\beta$ and IL-6, and it is characterized by secretion of IL-17 (figure 3). It is thought that Th17 cells protect against extracellular bacteria, particularly in the gut (Schoenborn \& Wilson, 2007), however they have also been extensively associated with autoimmune diseases (McGeachy \& Cua, 2008). Another type of $\mathrm{CD}^{+} \mathrm{T}$ cells consists on regulatory $\mathrm{T}$ cells (Tregs), which express the transcription factor Foxp3, secrete mainly IL-10 and suppress several of the effector functions of Ag-specific T cells (Nouri-Shirazi et al., 2000) (figure 3). Due to their inhibitory properties, an exacerbated activity of Tregs could impair $\mathrm{T}$ cell immunity and promote tumor development or susceptibility to infections. In contrast, a deficient activity of Tregs could lead to the loss of tolerance to self-Ags and thus promote autoimmune responses.

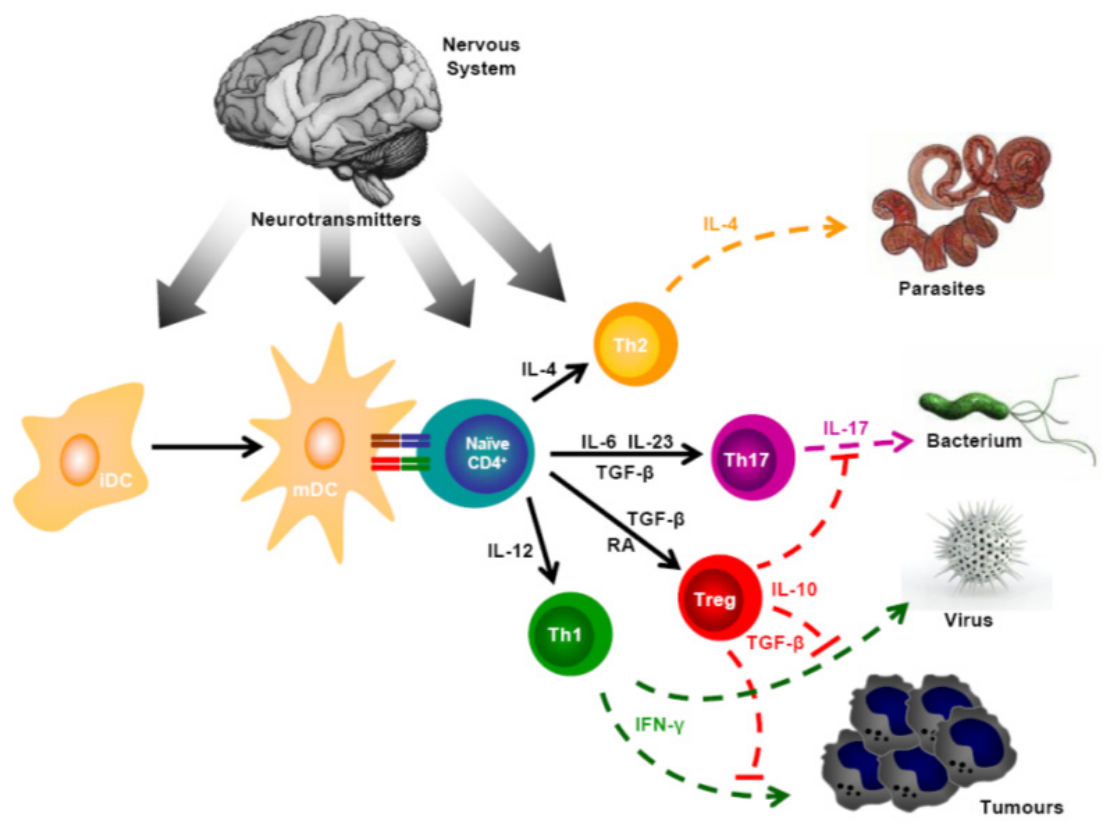

Figure 3. Regulation of immunity by neurotransmitters. DCs contribute to the acquisition of the most appropriate effector phenotype of $\mathrm{CD}^{+} \mathrm{T}$ cells, a process that can be regulated by neurotransmitters at several levels, such as DCs maturation, T cell activation and differentiation. 


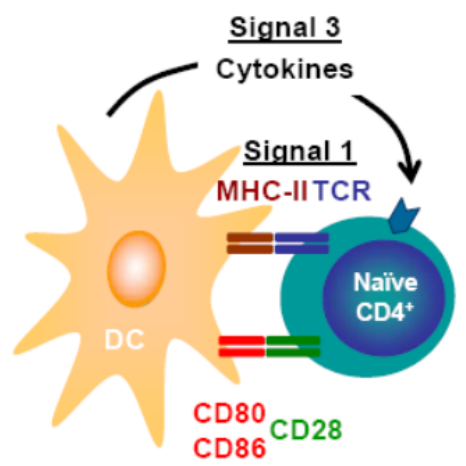

Signal 2

Figure 4. Three kinds of signals are involved in the activation and differentiation of $\mathrm{T}$ cells upon their interaction with DCs. The TCR/pMHC interaction determines the specificity of Ag recognition (signal 1). A second complementary signal corresponds to membrane-bound costimulatory molecules, such as CD80 and CD86, which determine the immunogenicity of DCs (signal 2). Finally, soluble molecules secreted by DCs (signal 3) may modulate the acquisition of different functional phenotypes by stimulating their receptors expressed on the $\mathrm{CD}^{+} \mathrm{T}$ cell surface.

DCs are the most potent APCs specialized in the initiation of adaptive immune responses by directing the activation and differentiation of naïve T cells (Lanzavecchia \& Sallusto, 2001). These cells can capture both self and foreign Ags in diverse tissues and migrate to secondary lymphoid organs, such as lymph nodes or spleen, to present processed Ags on MHC molecules to T cells. DC-T cell interaction can control and regulate $\mathrm{T}$ cell activation, the polarization of the effector phenotype, and the induction of tolerance (Friedl et al., 2005). Important functional components of the DC-T cell synapse are soluble molecules as well as membrane-bound receptors pairs expressed either on the DC or the T cell surface. The most significant intercellular molecular interactions mediating DC-T cell communications are $\mathrm{TCR} / \mathrm{pMHC}$ interaction, costimulatory molecules with their corresponding receptors and cytokines with their associated receptors. The TCR/pMHC interaction determines the specificity of $\mathrm{T}$ cell activation (signal 1, see figure 4). Recognition of pMHC by the TCR is required to trigger all of the later signaling events occurring during $\mathrm{T}$ cell function. When Ags are captured by DCs in an inflammatory context generated by cell damage, inflammatory cytokines or pathogen-associated molecular patterns, DCs undergo a maturation process consisting on the expression of elevated levels of costimulatory molecules. CD80 and CD86 (Zheng et al., 2004) are two membrane-bound costimulatory molecules that contribute to the immunogenicity of DCs. Both CD80 and CD86 can bind to CD28 on $\mathrm{T}$ cells providing costimulatory signals, which are complementary to TCRtriggered signaling and promote efficient $\mathrm{T}$ cell activation (signal 2, see figure 4). The differential expression of these costimulatory surface molecules allows DCs to modulate the nature of $\mathrm{T}$ cell responses, promoting either immunity or tolerance, respectively. There is also an important system of soluble proteins and their associated membrane receptors by which DCs regulate the outcome of immune responses. These soluble molecules correspond to a group of regulatory cytokines secreted by DCs, which by stimulating their receptors 
expressed on the $\mathrm{CD}^{+} \mathrm{T}$ cell surface, modulate the acquisition of different functional phenotypes such as Th1, Th2, Th17 and Tregs, as commented above (see figure 3). Consequently, by a coordinate expression of surface and soluble molecules, DCs can either define the nature of an adaptive immune response against a particular $\mathrm{Ag}$ or induce tolerance to self constituents (figure 4). Therefore, DCs are critical and highly relevant at promoting immunity against pathogens and tumors, avoiding the development of autoimmune damage to self healthy tissues.

\subsection{Nervous system mediated regulation of immunity}

Traditionally, it has been described that the function of immune cells, such as $\mathrm{T}$ cells and DCs, is regulated by soluble protein mediators known as cytokines. However, an emerging number of studies have shown that immune system cells can be also regulated by neurotransmitters (Franco et al., 2007). In this regard, it has been described that several receptors for neurotransmitters classically expressed in the nervous system, are also expressed on the surface of immune system cells. For instance, T cells and DCs express some subtypes of glutamate receptors (GluRs), acetylcholine receptors (AChRs), serotonin receptors (5-HTRs), dopamine receptors (DARs), adrenergic receptors, and others (Pacheco et al., 2010). Similar to T cells and DCs, neurotransmitter receptors have been found on the surface of a number of other immune system cells, which implicates that these cells could be regulated by molecules from the nervous system. The identification of these receptors on immune system cells suggests that neurotransmitters play a physiological role in the regulation of the immune response. Thus, deregulation on the activation of these receptors could be involved on the development of autoimmunity or cancer. Moreover, this fact implicates that different physiological or pathological states of the nervous system could be involved in the regulation of immune response. Due to the fact that DCs and T cells express receptors for neurotransmitters present in both compartments, the central nervous system (CNS) and the peripheral nervous system (PNS), immune response can be influenced by these two different parts of the nervous system. In the next sections, some examples of neurotransmitter-mediated regulation of DCs and T cells function (section 2.2.1) as well as the regulation of immunity by the PNS (section 2.2.2) will be analyzed. The interaction of immune cells with the CNS will be analyzed later, in section 2.3, together with neurodegenerative diseases that involve adaptive immune responses inside the CNS.

\subsubsection{Neurotransmitter mediated regulation of T cell and DC physiology}

By stimulating different neurotransmitter receptors expressed on $\mathrm{T}$ cells or DCs, neurotransmitters from diverse sources may strongly regulate the initiation and development of immune responses. Although at low levels, some neurotransmitters can be found in plasma and altered concentrations may reflect different physiological states of the nervous system. Thus, neurotransmitters present in plasma may constitute the primary source of these molecules for immune cells that are found migrating through the blood, such as patrolling naïve or memory $\mathrm{T}$ cells or effector $\mathrm{T}$ cells migrating toward the place of infection. The existence of innervation of lymphoid tissues by the autonomic nervous system 
represents an important source of neurotransmitters for immune cells. Innervation of primary lymphoid organs, such as bone marrow and thymus, could regulate the generation and differentiation of new lymphoid and myeloid cells by the nervous system, however, currently, there are not conclusive evidences on this area. On the other hand, the innervation of secondary lymphoid tissues, such as lymph nodes and spleen, by the autonomic nervous system constitutes a very relevant source of neurotransmitters for immune cells (Mignini et al., 2003). Importantly, Ag-presentation and T cell activation occurs in secondary lymphoid tissues, thereby innervation of these organs may represent the main nervous systemmediated regulation of initiation and development of the immune response. Finally, another way by which nervous system may regulate immune cells function through neurotransmitters release is when immune cells, including T cells and DCs, cross the bloodbrain-barrier (BBB) and enter into the CNS. Details of how immune cells enter into the CNS are discussed later, in section 2.3. Due to the key role played by $\mathrm{T}$ cells and DCs during adaptive immune responses, the contribution of some neurotransmitters in the function of these cells is here analyzed. Specifically, we have focussed the discussion on the involvement of dopamine and acetylcholine in the function of T cells and DCs and in the interaction between these two important immune cells (figure 5).
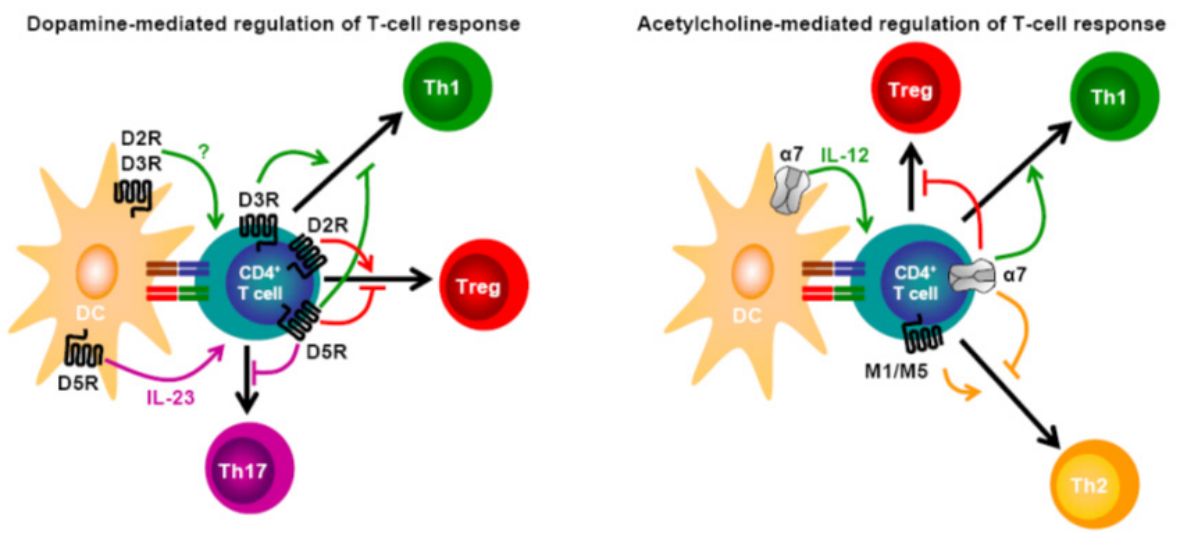

Figure 5. Neurotransmitter-mediated regulation of T cell response. Left panel, stimulation of D5R expressed on DCs promotes secretion of IL-23 favoring Th17 differentiation; while selective stimulation of D2R/D3R on DCs favors polarization of $\mathrm{CD} 4^{+} \mathrm{T}$ cell responses toward Th1. On the other hand, stimulation of D3R expressed on $\mathrm{CD} 44^{+} \mathrm{T}$ cells promotes $\mathrm{Th} 1$ differentiation, whereas $\mathrm{D} 2 \mathrm{R}$ stimulation on these cells would facilitate differentiation to Tregs. Finally, stimulation of D5R expressed on CD4 $4^{+} \mathrm{T}$ cell inhibits Th1 and Th17 differentiation, and Tregs function. Right panel, stimulation of $\alpha 7 \mathrm{nAChRs}$ expressed on DCs potentiates secretion of IL-12, improving anti-tumor response in vivo. Stimulation of M1/M5 mAChRs expressed on CD4 $4^{+} \mathrm{T}$ cells potentiates Th2 responses, while stimulation of $\alpha 7 \mathrm{nAChRs}$ expressed on $\mathrm{CD} 4^{+} \mathrm{T}$ cells favors Th1 responses and impairs Th2 and Tregs differentiation.

The first example here is dopamine-mediated regulation of immunity. So far, five DARs have been described, all of which are heptaspanning-membrane-receptors that belong to the superfamily of $G$ protein-coupled receptors. All five DARs have been described to be expressed on T cells. In general, the D3R receptor promotes stimulating functions in T cells, 
while D1R, D2R, D4R and D5R induce inhibitory signals that suppress the T cell function. D3R-stimulation induces secretion of IFN- $\gamma$ and inhibition of IL-10 and IL-4 synthesis in both $\mathrm{CD}^{+}$and $\mathrm{CD} 8^{+} \mathrm{T}$ cells, which suggests that D3R would favor a Th1-like response. In addition, D3R-triggered signaling not only stimulates chemotaxis by itself, but also potentiates migration of $\mathrm{CD}^{+} \mathrm{T}$ cells induced by some chemokines. On the other hand, stimulation of D1R/D5R impairs proliferation and cytotoxic effector function of CD4 ${ }^{+}$and $\mathrm{CD}^{+} \mathrm{T}$ cells, respectively, as well as inhibits Tregs function. D2R stimulation promotes enhanced production of IL-10, a cytokine that negatively regulates the function of effector T cells and it could be involved in the polarization toward Tregs. Stimulation of dopamine $\mathrm{D} 4 \mathrm{R}$ induces $\mathrm{T}$ cell quiescence, a state characterized by decreased cell size and metabolic activity of $\mathrm{T}$ cells. In addition to the different effects of DARs stimulation in the $\mathrm{T}$ cell physiology, it is important to consider that each DAR display different affinity for dopamine, having higher affinity D3R $>$ D5R $>$ D4R $>$ D2R $>$ D1R $(K i(n M)=27,228,450$, 1705,2340 , respectively). Thus, low levels of dopamine (e.g. $50 \mathrm{nM}$ ) would stimulate mainly D3R in $\mathrm{T}$ cells, favoring Th1-like responses and T-cell migration, whereas moderate dopamine levels (e.g. $300 \mathrm{nM}$ ) should stimulate D5R as well, inhibiting T cell function. It is expected that higher dopamine levels, by stimulating multiple DARs, would promote very complex effects in the $\mathrm{T}$ cell physiology, probably inhibiting $\mathrm{T}$ cell mediated immunity (Pacheco et al., 2009). With regard to the expression of DARs on DCs, D1R and D5R are the DARs expressed at higher levels on the cell surface, whereas D3R and D2R are poorly represented on the DCs surface. It has recently been demonstrated that stimulation of D5R on DCs strongly potentiates production of IL-23, a regulatory cytokine that favors polarization of naïve $\mathrm{T}$ cells toward the inflammatory Th17 phenotype. In fact, it was demonstrated that stimulation of D5R on DCs potentiates Th17 responses in vitro and in vivo (Prado et al., 2012). Other studies have shown that selective stimulation of D2R/D3R or selective inhibition of D1R/D5R on DCs favors polarization of $\mathrm{CD}^{+} \mathrm{T}$ cell responses toward Th1 and impairs the Th17 fate. Thus, depending on the concentration of dopamine, the specific DARs expressed and the kind of immune cell bearing DARs in the place where dopamine is available, this neurotransmitter may induce different effects in the immune response (figure 5, left panel).

The second example is the regulation of $\mathrm{T}$ cell responses by the neurotransmitter acetylcholine (Pacheco et al., 2010). This neurotransmitter may promote diverse effects on target cells by stimulating acetylcholine receptors (AChRs) expressed on the surface of those cells. AChRs described are classified in two main groups; namely nicotinic AChRs (nAChRs), which form ion channels and mediate fast excitatory acetylcholine responses, and muscarinic AChRs (mAChRs), which are heptaspanning-membrane-receptors and belong to the superfamily of $G$ protein-coupled receptors. Dopamine, glutamate, serotonin and other neurotransmitters constitute a group of physicochemically and enzymatically stable molecules, which may act on target cells relatively far from where these neurotransmitters were originally released (volume transmission). Thus, substantial amounts of these neurotransmitters can be detected in extracellular fluids including plasma and their role as neurotransmitters is terminated mainly by reuptake into nerve terminals and tissues. In contrast, acetylcholine belongs to the group of labile compounds which, when released, 
achieve effective concentrations to act over target cells for less than several milliseconds due to its rapid degradation by cholinesterases that are abundant in tissues and plasma (Franco et al., 2007). Thereby, very low acetylcholine levels are detected in plasma and it seems likely that this neurotransmitter primarily acts over immune system by wiring transmission between closely intercellular interactions. Regarding AChRs expression on T cells, it has been described that M3 is the most strongly expressed mAChRs followed by M5 and M4, whereas that M1 and M2 expression is substantially variable among individuals. Muscarinic stimulation on $\mathrm{T}$ cells promotes potentiation of $\mathrm{T}$ cell activation with strong IL-2 production which is mediated by the M3 subtype. Furthermore, is has been demonstrated that stimulation of the M1 subtype of $\mathrm{mAChRs}$ is not required for initial $\mathrm{T}$ cell activation, but for subsequent differentiation from naïve $\mathrm{CD} 8^{+} \mathrm{T}$ cells toward effector cytotoxic $\mathrm{CD} 8^{+} \mathrm{T}$ cells. In addition, studies suggest that stimulation of M1/M5 mAChRs expressed on CD4 ${ }^{+} \mathrm{T}$ cells potentiates Th2 responses in vivo. With regard to the function of nAChRs on T cells, it has been demonstrated that nicotine, mainly through $\alpha 7$ subunit-bearing $\mathrm{nAChRs}$, triggers intracellular signals. Currently, evidence suggests that stimulation of nAChRs $\alpha 7$ on T cells favors Th1 responses, whereas impairs Th2 and Tregs differentiation. Expression of nicotinic as well as muscarinic AChRs has also been described in DCs. However, consequences in DCs physiology upon stimulation of these AChRs have been poorly studied. In this regard, it has been demonstrated that targeting nAChRs $\alpha 7$ on DCs ex vivo results in increased IL-12 secretion, which in turn, induces enhanced stimulation of cytotoxic CD8 ${ }^{+} \mathrm{T}$ cells with a consequent improvement of anti-tumor response in vivo. These results suggest that stimulation of nAChRs $\alpha 7$ on DCs favors a pro-Th1 phenotype which stimulates polarization of naïve $\mathrm{T}$ cells toward Th1. Similar to what happen with dopamine and with other examples of neurotransmitters not mentioned here, depending on the local concentration reached, specific receptors expressed on immune cells, the kind of immune cells located in the microenvironment where acetylcholine is released, this neurotransmitter may exert different and complex regulation of $\mathrm{T}$ cell responses (figure 5, right panel).

Finally, as a conclusion in this section, several neurotransmitters may modulate $\mathrm{T}$ cell response by stimulating neurotransmitter receptors directly on $\mathrm{T}$ cells or by stimulating their receptors on DCs. Consequent neurotransmitter-mediated modulation may favor polarization of the $\mathrm{T}$ cell response toward different fates, such as Th1, Th17 or Tregs. Neurotransmitters that induce Th1 responses normally favor anti-viral, anti-tumor and antiintracellular bacterium response, whereas neurotransmitter contributing to promote strong Th17 and decreased Tregs function may be detrimental in the scenario of autoimmunity. Thus, imbalance on neurotransmitter-mediated regulation of immunity may play an important role in pathologies.

\subsubsection{Peripheral nervous system-mediated regulation of immunity}

Several studies performed mainly during the last decade have demonstrated a direct regulation of $\mathrm{T}$ cell responses by the sympathetic nervous system (SNS). These studies were mainly carried out administering 6-hydroxydopamine, a neurotoxic drug that selectively ablates noradrenergic and dopaminergic neurons, as this molecule is captured specifically 
through dopamine transporters (DAT) or norepinephrine transporters (NET). Due to the fact that 6-hydroxydopamine cannot cross the BBB, this drug depletes SNS without affecting neurons in the CNS when administered systemically. Norepinephrine is the main neurotransmitter released by the SNS and therefore this is probably the main neurotransmitter responsible for SNS-mediated regulation of immunity, however dopamine has also been involved in the SNS-mediated regulation of T cell response. The evidences point to a dual role of SNS on T cell responses. First, the SNS, probably by releasing norepinephrine, inhibits TGF- $\beta$ production in the spleen and lymph nodes, thus attenuating generation and function of Tregs. The relevance of this mechanism is evidenced in autoimmunity, as arthritis and experimental autoimmune encephalomyelitis (EAE) are developed with significant less severity in sympathectomized mice than in animals bearing intact SNS (Harle et al., 2008; Bhowmick et al., 2009). Second, by stimulating $\beta 2$-adrenergic receptors, SNS decreases immunogenicity of APCs in secondary lymphoid organs, attenuating both the Th1 response and the $\mathrm{CD} 8^{+} \mathrm{T}$ cell mediated cytotoxic activity. The relevance of this mechanism has been demonstrated in two models of infection: influenza and Listeria monocytogenes. Efficient clearance of these infectious agents requires potent Th1 and cytotoxic responses. In both cases, sympathectomized mice mounted a stronger antiviral and antibacterial response than those mice with intact SNS (Miura et al., 2001; Grebe et al., 2009). In addition, it is likely that anti-tumor immune response would be strengthened in sympatectomized mice, as this response requires Th1 polarization (see figure 3 ). The fact that in the first group of studies here described, the SNS potentiates autoimmune responses while in the second group, the SNS attenuates antiviral and antibacterial responses could be explained by a mechanism in which SNS-mediated attenuation of Th1 responses concomitantly favors Th17 responses. However, this possibility or another explanation for these observations is still pending to be clarified. The integrated mechanism mediating SNSinduced regulation of $\mathrm{T}$ cell response is schematized in figure 6. Another mechanism that could be relevant in SNS-mediated regulation of immunity involves dopamine. SNS neurons express the enzyme dopamine- $\beta$-hydroxylase $(\mathrm{D} \beta \mathrm{H})$, which is necessary to synthesize norepinephrine from dopamine. Dopamine is normally present in low amounts in SNS neurons in normal mice. However, in dopamine- $\beta$-hydroxylase deficient mice, all noradrenergic neurons become exclusively dopaminergic. It has been shown that $\mathrm{D} \beta \mathrm{H}$ deficient mice develop an attenuated antibacterial response against Listeria monocytogenes, when compared with normal mice (Alaniz et al., 1999). Thus, exacerbated dopamine signaling by the SNS results in a decreased Th1 response.

Another PNS-mediated regulation of immunity is the well-documented cholinergic antiinflammatory pathway, also known as the inflammatory reflex, which results in attenuated activation of splenic macrophages. This pathway does not constitute a nervous system mediated regulation of $\mathrm{T}$ cell responses, however, this pathway use $\mathrm{T}$ cells as intermediate cells to control innate immunity. Detection of pathogen- or damage- associated molecular patterns by specialized receptors expressed on macrophages, results in a strong production of pro-inflammatory cytokines, such as TNF- $\alpha$, IL-6, IL-8 and IL-1. When elevated levels of these cytokines are produced, they are transported throughout the blood and detected in the brain as a signal of excessive inflammation. Consequently, the nervous system 
responds with the inflammatory reflex, which is triggered as action potentials transmitted through the vagus nerve. Outflows from vagus nerve arrive at the coeliac ganglion, which subsequently triggers action potentials in the splenic nerve. Outflows arrive to the spleen, where, by a mechanism dependent on nAChR $\alpha 7$, attenuate the production of inflammatory cytokines by splenic macrophages (Rosas-Ballina \& Tracey, 2009). Paradoxically, nerve fibers in spleen, originating in the coeliac ganglion, are noradrenergic, not cholinergic, and utilize norepinephrine as the main neurotransmitter. Recently, it was demonstrated that norepinephrine released from splenic nerve stimulates $\beta 1$ - and $\beta 2$ adrenergic receptors on memory $\mathrm{T}$ cells, which consequently release high amounts of acetylcholine (Rosas-Ballina et al., 2011). Acetylcholine derived from $\mathrm{T}$ cells acts subsequently on $\mathrm{nAChR} \alpha 7$ expressed on macrophages triggering inhibitory signals that limit inflammatory cytokine release and thus, maintain homeostasis (figure 7). Regarding the sympathetic or parasympathetic origin of this PNS-mediated mechanism, it actually cannot be classified as any of them, as classic parasympathetic signals in the periphery are transduced by the vagus nerve and by mAChRs, not nAChRs. This mechanism is relevant and conceptually very interesting because, first, $\mathrm{T}$ cells act as a regulatory bridge between nervous system and innate immunity and, second, $\mathrm{T}$ cells work as reservoirs of neurotransmitters, releasing them under specific stimuli, which act subsequently over another immune system cells. This latter concept will be developed in more detail later in this chapter, in section 3.1.

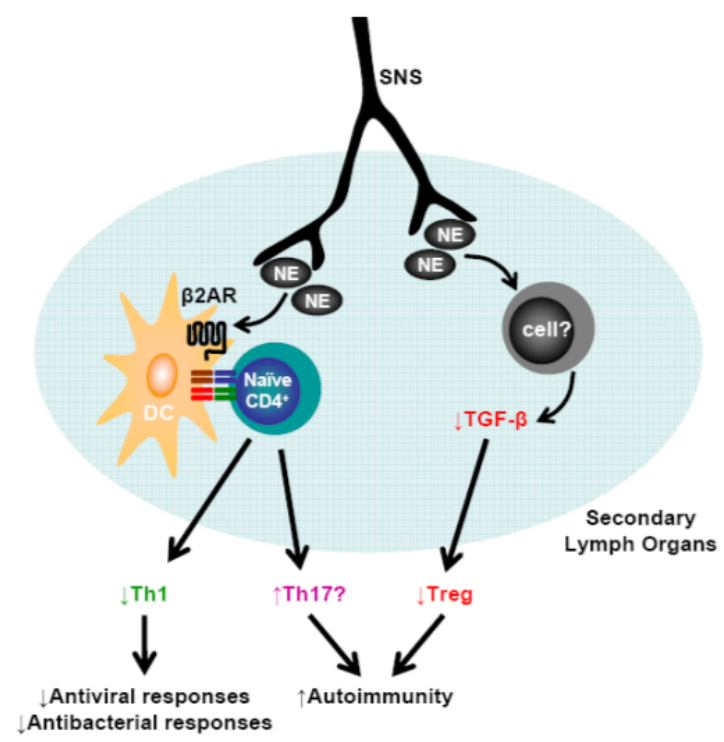

Figure 6. SNS-mediated regulation of T cell response. For explanation see the text. NE, Norepinephrine; $\beta 2 \mathrm{AR}, \beta 2$-adrenergic receptors. 


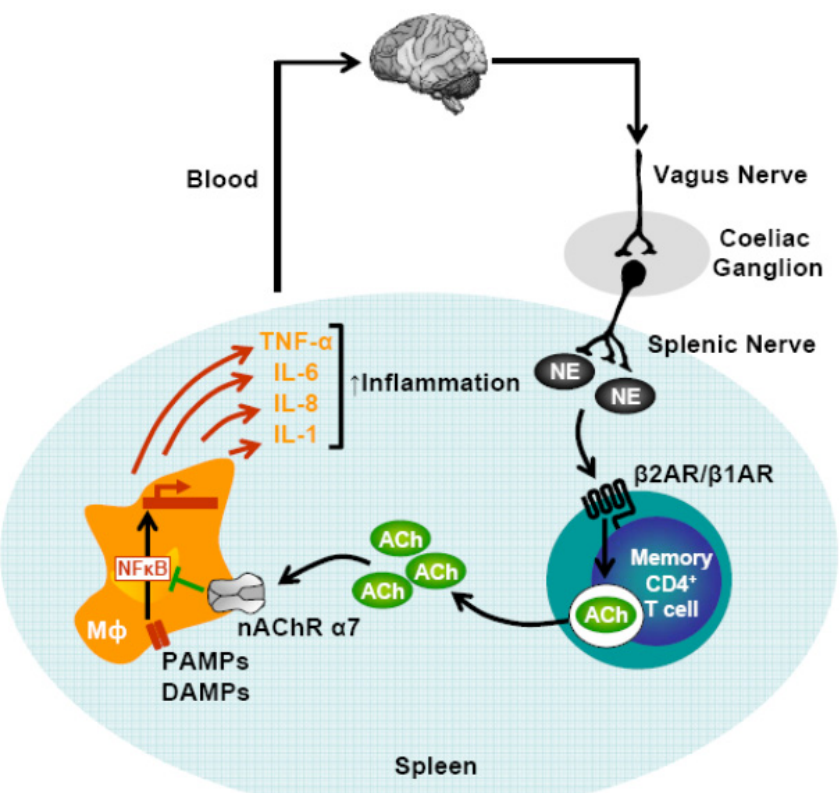

Figure 7. SNS-mediated regulation of innate immunity. Pathogen or damage recognition by spleen macrophages triggers strong production of pro-inflammatory cytokines. Elevated levels of inflammatory cytokines in blood are detected by the nervous system triggering the inflammatory reflex. For explanation see the text. NE, Norepinephrine; DAMPs, Damage-Associated Molecular Patterns; PAMPs, Pathogen-Associated Molecular Patterns.

\subsection{Immune system mediated regulation of the nervous system}

Entrance of immune system cells in the parenchyma of CNS is restricted, primarily because of the BBB that surrounds parenchymal venules. This barrier is constituted by a first layer of specialized endothelial cells of the blood vessel wall, which are intimately connected by tight junctions. This first layer of cells is surrounded by a basement membrane, and an outer layer constituted by astrocyte feet and microglial cells. Under normal physiological conditions no immune cells are found in the CNS parenchyma. However, some immune cells, including lymphoid and myeloid cells, may infiltrate into the cerebrospinal fluid, which is produced by choroid plexus epithelia and flows throughout the space comprised between the meninges, the subarachnoid space. Importantly, cerebrospinal fluid drains into cervical lymph nodes, enabling peripheral immune cells to survey and respond to CNS Ags under certain pathological conditions. Thus, although the access to the CNS parenchyma of the healthy brain is restricted for immune cells, these cells can enter into the cerebrospinal fluid through choroid plexus. Accordingly, subarachnoid space and choroid plexus of healthy mice are populated by substantial numbers of $\mathrm{T}$ cells and heavily populated by myeloid cells. In this regard, DCs that derive from local precursors and exhibit a differentiation and Ag-presenting program similar to spleen DCs, have been identified in 
the brain of healthy mice (Anandasabapathy et al., 2011). Importantly, cells of the immune system not only have been related with surveillance in the CNS. Under normal conditions, immune mechanisms are activated by environmental/psychological stimuli and they positively regulate the remodeling of neural circuits, promoting memory consolidation, hippocampal long-term potentiation (LTP) and neurogenesis (Yirmiya \& Goshen, 2011). According to this, recent studies have demonstrated that spatial memory and learning are $\mathrm{CD}^{+} \mathrm{T}$ cell-dependent in healthy animals. In particular, IL-4-producing $\mathrm{T}$ cells accumulate in the subarachnoid space during cognitive tasks. IL-4 produced by these Th2 cells in turn stimulates hippocampal astrocytes to produce and release brain-derived-neurotrophic-factor (BDNF), which acts subsequently on hippocampal neurons (figure 8, left panel), favoring spatial-memory and learning (Derecki et al., 2010). These authors have demonstrated an impaired learning in mice lacking $\mathrm{CD}^{+}{ }^{+} \mathrm{T}$ cell or in mice bearing IL-4-deficient CD4+ T-cells. In both cases, normal learning is recovered when mice are reconstituted with normal $\mathrm{CD} 4^{+} \mathrm{T}$ cell, thus linking immune activity to steady-state cognitive function. The importance of $\mathrm{T}$ cells in cognitive function is also supported by more common or natural conditions such as aging, HIV infection and chemotherapy. All of these conditions are associated with decreased $\mathrm{T}$ cell function which is accompanied by cognitive impairments (Yirmiya \& Goshen, 2011).

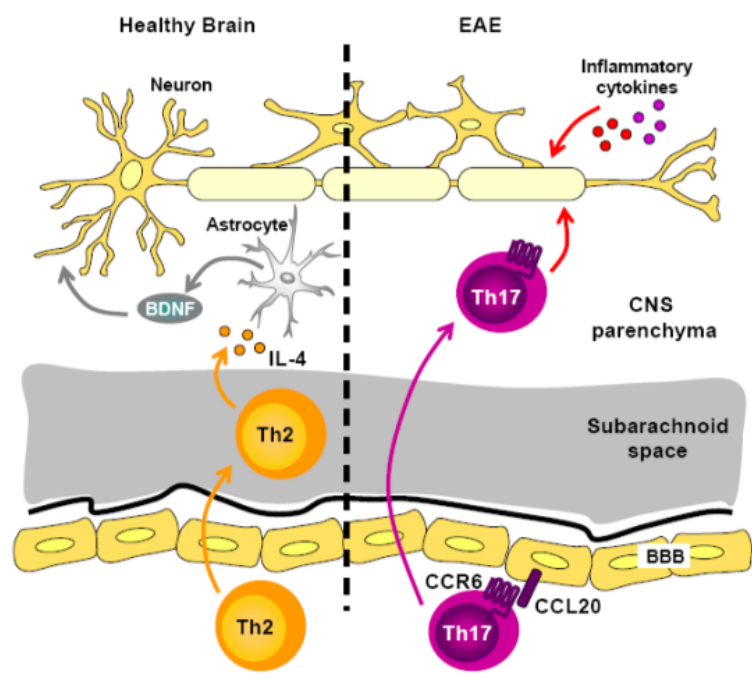

Figure 8. T cell-mediated regulation of the CNS activity. Left panel, in healthy brain, Th2 cells accumulate in the subarachnoid space during cognitive tasks and secrete IL-4, which diffuses into the parenchyma and stimulates astroglia. Consequently, IL-4-stimulated astroglia produces BDNF, which acts on hippocampal neurons favoring acquisition of spatial memory and learning. Right panel, in an autoimmune disease directed against CNS-derived Ags such as EAE, choroid plexus express the chemokine CCL20, which allows CCR6-expressing Th17 cells to cross the BBB and to be recruited into the CNS parenchyma. Subsequently, these Th17 cells mediate the destruction of myelin sheath and axonal damage by the secretion of proinflammatory cytokines and the recruitment of innate immune cells. 
On the other hand, the presence of infiltrating immune cells into the CNS parenchyma has been detected in most of the neurodegenerative diseases studied. In a pathological scenario involving the CNS, such as neurodegeneration or imbalance of glial homeostasis, initial neuroinflammatory processes induce brain endothelial cells to express a specialized pattern of adhesion molecules. Adhesion molecules induced by inflammatory processes subsequently allow activated $\mathrm{T}$ cells to adhere to vessel walls and to be recruited into the CNS parenchyma. T cells that infiltrate the CNS are previously activated in the periphery, presumably in cervical lymph nodes, in which a sampling of CNS-associated Ags is constantly arriving through the cerebrospinal fluid that drains these lymph nodes. Interestingly, the expression of the chemokine receptor CCR6 by a subset of pathogenic T cells, Th17 cells, and the expression of its corresponding ligand, the chemokine CCL20, by epithelial cells of the choroid plexus have been shown to play an important role in facilitating Th17 cells recruitment to BBB during the development of EAE, the murine model of the autoimmune disease multiple sclerosis. It has been determined that IL-17R and IL-22R are up-regulated on the surface of endothelial cells of BBB in multiple sclerosis lesions. Stimulation of these receptors by IL-17 and IL-22 secreted by Th17 cells induces disruption of tight junctions. Thus, Th17 cells perform a particular and specialized mechanism to cross the BBB (Pacheco et al., 2009). Th17 cells infiltrated into CNS parenchyma interact with microglia, promoting the production of additional inflammatory molecules that up-regulate some key adhesion molecules and chemokines that, in turn, allow subsequent recruitment of another kind of leukocytes through BBB, including Th1 cells and DCs. Once in CNS parenchyma, infiltrating $\mathrm{T}$ cells can contribute significantly to regulate the neurodegenerative process by the secretion of different cytokines and the recruitment of innate immune cells. In the case of multiple sclerosis or EAE, CNS-infiltrating T cell acquire aggressive phenotypes and recruit effector cells from the innate immune system which play an important role in mediating demyelination and axonal damage (figure 8, right panel) (Goverman, 2009). Additionally, molecules derived from immune cells can act over glial cells, modulating microglia-mediated neurotoxicity. In this regard, recent studies have shown that peripheral $\mathrm{T}$ cells infiltrate into the brain parenchyma at the site of neuronal injury in Parkinson's disease. This $\mathrm{T}$ cell-mediated immune response contributes significantly to the destruction of dopaminergic neurons, through a CD4 ${ }^{+} \mathrm{T}$ cell-dependent cytotoxic mechanism. These studies support the involvement of pathogenic $\mathrm{CD} 4^{+} \mathrm{T}$ cell populations, which would induce acquisition of an M1-like pro-inflammatory phenotype by microglia, which is characterized by the secretion of inflammatory factors, such as TNF- $\alpha$, IL-1 $\beta$ and superoxide. Conversely, in the healthy scenario, other T cell subsets, for instance Tregs and Th2, could contribute to microglial acquisition of an M2-like anti-inflammatory phenotype, which release neurotrophic factors, such as IGF-1 (figure 9), promoting neuronal protection (Appel, 2009; Reynolds et al., 2010). In opposition to the role of CNS-infiltrating T cells in Parkinson's disease and multiple sclerosis, studies carried out in amyotrophic lateral sclerosis have shown that absence of $\mathrm{T}$ cells accelerate motoneuron disease while the adoptive transfer of T cells ameliorates disease severity (Lucin \& Wyss-Coray, 2009). These results probably could be explained by the contribution of $\mathrm{T}$ cells to the acquisition of $\mathrm{M} 2$ phenotype by microglia, however further investigation is necessary to confirm this 
hypothesis. Thus, imbalance on these different kinds of neuro-immune interactions may favor the onset of autoimmune disorders and also may constitute an important component of the pathogenic mechanisms involved in neurodegenerative diseases.

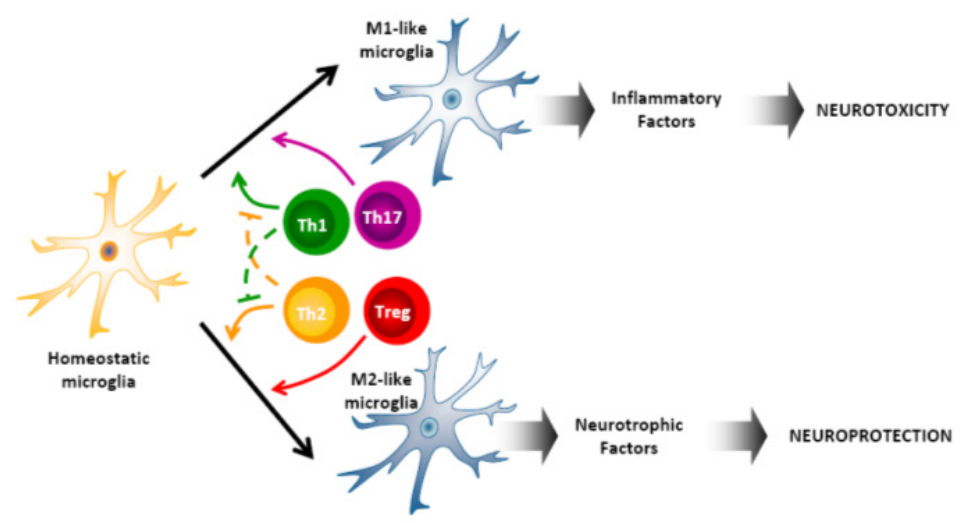

Figure 9. Involvement of $\mathrm{CD} 4{ }^{+} \mathrm{T}$ cells in the microglia-mediated neurotoxicity or neuroprotection. By recognizing pathogen- or damage- associated molecular patterns, homeostatic microglia undergoes an activation process, which may result in the acquisition of two different phenotypes. The first one is the M1-like phenotype, which produces and releases inflammatory factors, thus promoting neuronal injury. The second one is the M2-like phenotype, which produces neurotrophic factors, sustaining neuroprotection. Different kinds of $\mathrm{CD}^{+} \mathrm{T}$ cell may differentially modulate acquisition of microglial phenotypes. Whereas Th1 and Th17 cells favor acquisition of M1-like phenotype by microglia, Th2 and Tregs contribute to the acquisition of M2-like phenotype. Th1 and Th2 cells may promote antagonistic effects in the acquisition of M2-like and M1-like phenotypes, respectively.

\section{The nervous system and immune system use the same mediator molecules}

For many years the immune system, including its cells and mediator molecules, was studied and analyzed completely separate from the nervous system and vice versa. Classically, cytokines have been considered the main soluble molecules mediating regulatory communications between immune cells. On the other hand, neurotransmitters have been described as the traditional soluble molecules mediating interactions between neurons or between glial cells and neurons. Currently, it is known that immune system cells can also communicate through neurotransmitters and that nervous system cells can communicate by cytokines as mediators. Evidences from the last decade have shown that immune cells not only express neurotransmitter receptors, but also have the capacity to synthesize and store neurotransmitters in intracellular compartments, which may be released under specific stimuli. Similarly, nervous system cells not only express cytokine receptors, but are also able to produce these molecules. This new knowledge has opened the possibility that immune cells could communicate between themselves through neurotransmitters and that nervous system cells could interact between themselves by cytokines (figure 10). In this chapter, first, the neurotransmitter-mediated communications between immune cells will be discussed 
(section 3.1); and second, cytokine-mediated interactions between nervous system cells will be also analyzed (section 3.2).

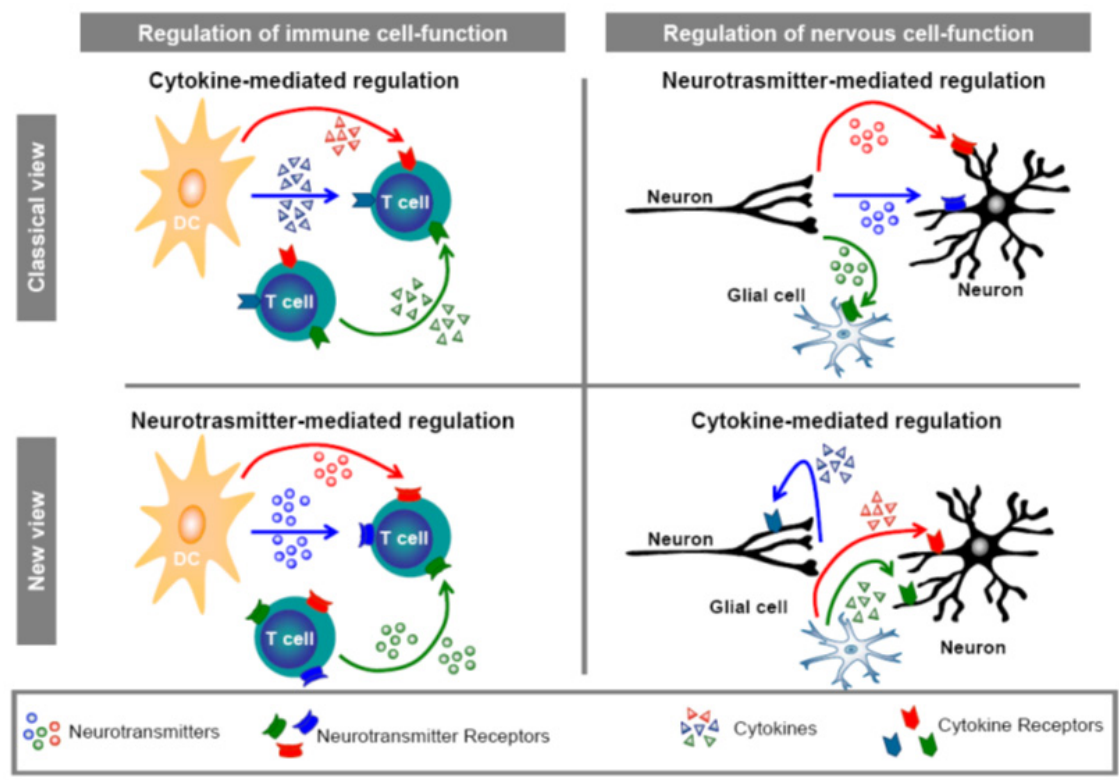

Figure 10. Immune and nervous systems utilize common modulatory molecules. The classical view states that immune system cells and nervous system cells communicate each other by means of cytokines and neurotransmitters, respectively. However, evidence from last decade supports the common usage of these molecules by both systems, resulting in neurotransmitter-mediated communication between immune cells and cytokine-mediated interaction among nervous system cells.

\subsection{Neurotransmitters as mediators of immune-mediated immunity}

An increasing number of studies have revealed that some cellular components involved in adaptive and innate immune responses such as DCs, T cells, B cells, macrophages and others, are capable of synthesizing and/or capturing classical neurotransmitters, including acetylcholine, dopamine, serotonin and glutamate. Under some stimuli, these cells may release neurotransmitters from vesicular or non-vesicular storages toward extracellular compartment, thus involving autocrine or paracrine communications with different leukocytes. For instance, $\mathrm{T}$ cells may release serotonin, norepinephrine, dopamine and acetylcholine, while DCs may release glutamate, dopamine and serotonin (Franco et al., 2007). This fact not only suggests that neurotransmitters could mediate communication between immune cells, but also that this kind of molecules may be involved in bidirectional cross-talk between immune and nervous system (figure 11). Next, some examples of neurotransmitter-mediated communications between immune cells are analyzed and discussed.

DCs capture and synthesize some neurotransmitters, which are stored in intracellular compartments and later used either to regulate their own functions (autocrine process) or to 
modulate T cells (paracrine process). Recently, it was demonstrated that DCs express the whole machinery to synthesize and store dopamine (Prado et al., 2012). For instance, these cells express tyrosine hydroxylase $(\mathrm{TH})$, which catalyzes the first step necessary for biosynthesis of dopamine. However, these cells do not express $\mathrm{D} \beta \mathrm{H}$, the enzyme necessary to metabolize dopamine and to transform it in epinephrine and norepinephrine. In addition, DCs do not express the plasma membrane dopamine transporter, necessary to take up dopamine from the extracellular compartment. Thereby, DCs synthesize dopamine, but not the other catecholamines, and they can not capture dopamine from the extracellular space. These cells also express enzymes necessary to degrade dopamine in the cytoplasm and vesicular transporters necessary to store dopamine in vesicular compartments. A recent study has indicated that LPS, a pathogen-associated molecular pattern, induces dopamine release from DCs, which subsequently acts in an autocrine manner stimulating D5R expressed on DCs (Prado et al., 2012). D5R stimulation promotes a potent production of IL23 by DCs, thus conferring these cells with the ability to promote polarization of $\mathrm{CD} 4{ }^{+}$naïve $\mathrm{T}$ cells toward the inflammatory Th17 phenotype (figure 12; see also figure 5, left panel). Therefore, this autocrine loop mechanism mediated by dopamine and stimulation of D5R on DCs, results to be one of the engines feeding autoimmune responses.

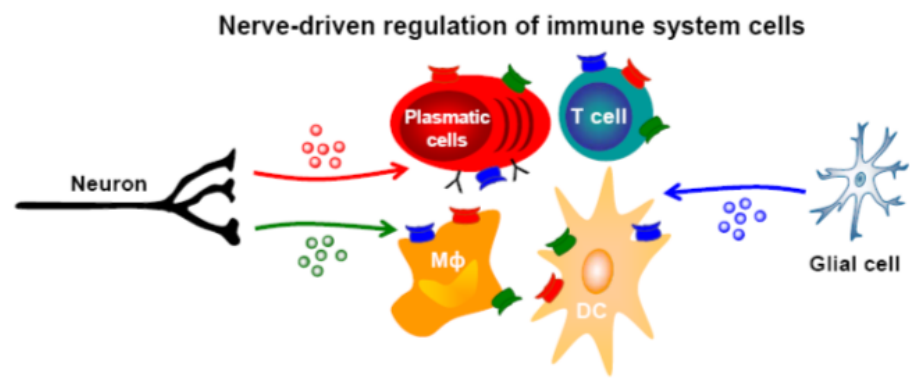

Immune-driven regulation of nervous system cells

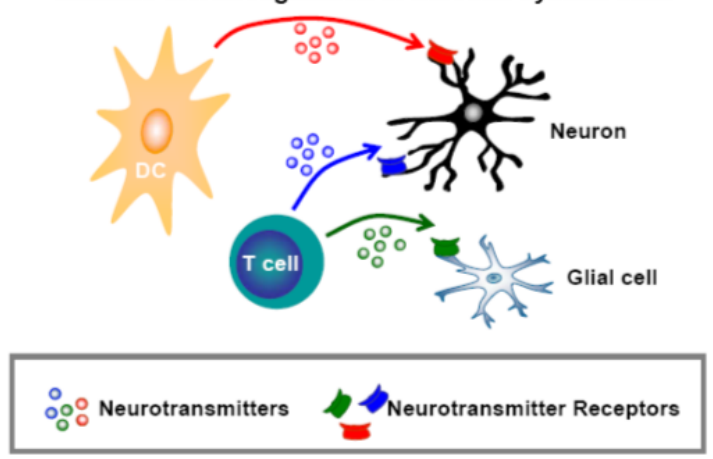

Figure 11. Neurotransmitters mediate bidirectional cross-talk between immune and nervous cells. Neurotransmitter-mediated inter-systemic communication may occur in both directions: the regulation of immune cells by nervous-derived neurotransmitters as well as the modulation of nervous cells by immune-derived neurotransmitters. Similarly, cytokine-mediated inter-systemic communication may occur in both directions, however it has been omitted in the figure for simplification. 


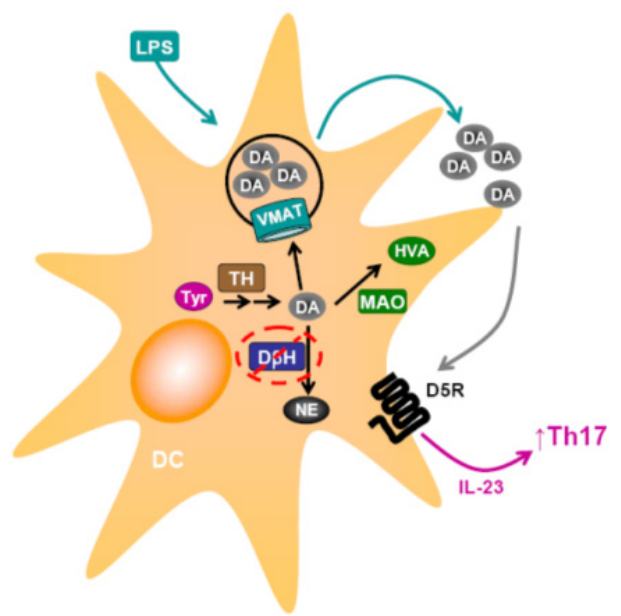

Figure 12. Dopamine-mediated regulation of $\mathrm{T}$ cell differentiation by DCs. VMAT, vesicular monoamine transporter; MAO, monoamine oxidase; DA, dopamine; NE, norepinephrine; HVA, homovanillic acid; Tyr, tyrosine. For explanation and other abbreviations see the text.

DCs may also represent a source of acetylcholine and serotonin for $\mathrm{T}$ cells (Franco et al., 2007; Pacheco et al., 2010). Acetylcholine released from DCs may constitute an important contribution during first stage of $\mathrm{T}$ cell activation. In this regard, it has been described that when undergoing maturation, DCs begin to express choline-acetyl-transferase (ChAT), the enzyme necessary to synthesize acetylcholine (Kawashima et al., 2007). These findings suggest that acetylcholine would be released during $\mathrm{Ag}$ presentation to $\mathrm{T}$ cells. Acetylcholine released during Ag presentation can, subsequently, exert different effects acting over AChRs expressed on T cells and on DCs (see figure 5, right panel). Regarding DCs as a source of serotonin, these cells do not synthesize this neurotransmitter but express the serotonin transporter (SERT) and therefore can efficiently sequester microenvironmental serotonin. Serotonin is stored in vesicles, which are released via $\mathrm{Ca}^{2+}$-dependent exocytosis during Ag-presentation. SERT expression by DCs is dynamically regulated. Thus, DCs appear to maximize their uptake and storage capacity for serotonin upon maturation or activation, prior to productive encounters with naïve $\mathrm{T}$ cells. In contrast, ligation of CD80/CD86 on DCs by the inhibitory ligand expressed on the T cell surface, the Cytotoxic TLymphocyte Antigen 4 (CTLA-4), downregulates SERT expression in DCs. Thus, the differential serotonin content of DCs may contribute to modify signaling at the T cell-DC interaction (see below).

Another kind of immune cells that represent a key source of neurotransmitters necessary to regulate immune responses are $\mathrm{T}$ cells. Accordingly, it has been described that Tregs constitutively express $\mathrm{TH}$ and contain substantial amounts of dopamine and other catecholamines, while effector $\mathrm{T}$ cells only contain trace amounts (Cosentino et al., 2007). Moreover, Tregs as well as effector T cells express vesicular monoamine transporters which allow these cells to accumulate catecholamines into vesicular storages. However, only Tregs 
store and release physiologically relevant amounts of dopamine from vesicular storages. The stimulus responsible for triggering dopamine release from Tregs is still unknown, however it is thought that dopamine from this source would constitute an autocrine loop in which, by stimulating D1R/D5R, this neurotransmitter inhibits the suppresive function of Tregs exerted over effector T cells (Kipnis et al., 2004; Cosentino et al., 2007).

Acetylcholine is also an important mediator produced and released by $\mathrm{T}$ cells. Indeed, it seems that this source of acetylcholine is the primary source of this mediator for these cells inside secondary lymphoid organs. All of the components of cholinergic system are expressed in T cells (Pacheco et al., 2010). In this regard, ChAT and acetylcholine stores have been found in memory CD4 ${ }^{+} \mathrm{T}$ cells (Rosas-Ballina et al., 2011). In addition to acetylcholine and ChAT, nAChRs, mAChRs, cholinesterase activity and choline transporters have also been found on the surface of these cells. Release of acetylcholine-containing vesicles from memory $\mathrm{CD}^{+} \mathrm{T}$ cells may be triggered by $\mathrm{T}$ cell activation or by norepinephrine derived from the PNS in the spleen (see figure 7) (Kawashima \& Fujii, 2003; Rosas-Ballina et al., 2011). Acetylcholine release may lead to high local concentrations of this neurotransmitter, which in turn can stimulate AChRs expressed on macrophages and in $\mathrm{CD}^{+} \mathrm{T}$ cells themselves. Stimulation of AChRs expressed on macrophages and on T cells may exert different effects depending on the acetylcholine concentration reached in the microenvironment (see figure $5 \mathrm{~B}$, right panel and figure 8 ).

Another example of neurotransmitter working as mediator of T cell-mediated regulation of immunity is serotonin (Franco et al., 2007; Pacheco et al., 2010). Splenic T cells selectively express tryptophan hydroxylase (TPH), the enzyme that catalyses conversion of L-tryptophan to 5-hydroxytryptophan, the immediate precursor of serotonin. Accordingly, $\mathrm{T}$ cells can synthesize serotonin and this capacity is considerably increased following activation. When $\mathrm{T}$ cells enter the activation process, serotonin release is induced. Thus, during Ag-presentation by DCs in secondary lymphoid organs, both T cells and DCs (see above) begin to release serotonin in the microenvironment of $\mathrm{T}$ cell-DC interaction. Serotonin seems to act early stimulating $5-\mathrm{HT}_{7}$ and $5-\mathrm{HT}_{3}$ receptors expressed on naïve T cells, potentiating $\mathrm{T}$ cell activation and proliferation in these cells. After initial activation, $\mathrm{T}$ cells begin to express $5-\mathrm{HT}_{1}$ and $5-\mathrm{HT}_{2}$ receptors which may promote further serotoninmediated regulation in the effector function of $\mathrm{T}$ cells, such as potentiating polarization of the effector phenotype acquired by T cells.

\subsection{Cytokines as mediators of nervous system-mediated nervous function}

Cytokines have been long recognized as peripheral mediators of inflammatory reactions, orchestrating complex immune responses against infection and injury. More recently, cytokines have been reported to be synthesized also in the brain by both the glial and the neuronal compartment. Moreover, cytokine receptors have also been found in different regions of the CNS. This section is focused first in the modulation of nervous function by glial cell-derived cytokines and then on the current evidence showing cytokine production by neurons. 
One of the normal processes recently found to be regulated by brain-derived cytokines is the LTP in the hippocampus, a mechanism characterized by a sustained enhancement in synaptic transmission following high frequency stimulation, and considered one of the main cellular mechanisms underlying learning and memory. Induction of LTP in hippocampal slice preparations and in freely moving rats caused a clear and persistent increase of IL-1 $\beta$ gene expression on glial cells. Induction of IL-1 $\beta$ production by glial cells requires previous release of neuregulin-1 (Nrg-1) by pre-synaptic neurons, a mediator that binds to its receptor erbB2 on the surface of surrounding glial cells, triggering thus IL-1 $\beta$ production (Ren \& Dubner, 2010). IL-1 $\beta$ in turn, stimulates its receptor on the post-synaptic neurons. IL1-induced signaling on these neurons enhances the capability of responses triggered via ionotropic NMDA glutamate receptor (figure 13). Thus, transient blockade of IL-1 receptors results in the inhibition of LTP maintenance. The key role of IL-1 in the acquisition of LTP is further supported by the complete absence of LTP observed in hippocampal slices obtained from IL-1 receptor knockout mice (Goshen et al., 2007). Moreover, the relevance of IL-1 in the acquisition of memory is supported by studies showing that lack of IL-1 signaling in the brain results in decreased performance on memory and learning tests, suggesting that IL-1mediated signaling in the hippocampus plays a critical role at establishing LTP and, consequently, learning and memory processes. In addition to its role in the establishment of beneficial processes, it was recently recognized that IL-1 $\beta$ expressed by glial cells may also have a detrimental role in pathology, such as in the genesis and maintenance of persistent pain. Several reports have suggested that IL-1 $\beta$ plays a central role in this process as direct injection of this cytokine into the CNS produces increased sensitivity to pain (hyperalgesia) and enhanced neuronal responses in animals. In line with this, the injection of Freund's adjuvant into the masseter muscle in rats, a model of persistent pain in which occurs muscle inflammation and hyperalgesia, results in increased IL-1 $\beta$ production by activated astroglia in the spinal trigeminal nucleus. On the other hand, evidence shows that IL-1 receptor is also co-expressed with NMDA receptor on post-synaptic neurons involved in pain persistance. Accordingly, it was shown that IL-1 receptor-mediated signaling increases NMDA receptor activity in post-synaptic neurons, increasing synaptic strength and contributing to inflammation-induced pain hypersensitivity. Thus, IL-1 $\beta$ provides a link between astroglial activation and NMDA receptor activation in neurons, a mechanism that results in increased memory acquisition and pain sensitivity.

Another cytokine involved in the regulation of nervous function is IL-6, which is also produced upon induction of LTP both in vitro and in vivo. However, in contrast to the supportive effect of IL-1 $\beta$, this cytokine contributes to the extinction of a consolidated LTP. In this regard, it has been shown that IL-6 neutralization after continuous stimulation of pre-synaptic neurons results in a marked prolongation of LTP, an effect that correlates with improved performance of animals in long-term memory tests. In agreement with this, IL-6 knockout mice also present enhanced learning capabilities compared to wild type littermates. This fact indicates that hippocampal expression of IL-6 limits the maintenance of LTP and, therefore, reduces memory and learning. Additional evidence has shown that IL-6 upregulation is tightly localized near the site of stimulation in LTP experiments and is detected only in non-neuronal cells (Besedovsky \& del Rey, 2011). This observation has prompted the current model of LTP modulation by 
cytokines, in which cytokine release by astroglial/microglial cells influence neuron function by acting either in a paracrine or an autocrine manner (figure 13).

Another example of cytokine operating as a modulator between nervous cells is TNF $\alpha$. In normal conditions, production of TNF $\alpha$ by glial cells enhances synaptic effects by increasing surface expression of ionotropic AMPA glutamate receptors on post-synaptic neurons (figure 13). In this regard, a decreased expression of AMPA receptors on post-synaptic neurons is observed when biological activity of this cytokine is prevented, suggesting that TNF $\alpha$ preserves synaptic strenght at excitatory synapses and that TNF $\alpha$-mediated signaling may play an important role in synaptic plasticity and in response to neural injury. Indeed, it has been shown that glia-derived TNF $\alpha$ plays a pivotal role in the development of synaptic scaling, one of the two mechanisms involved in the generation of synaptic plasticity, along with LTP, described to date (Stellwagen \& Malenka, 2006). In adittion, a recent report has identified neuronal TNF receptor subtype 1, but not 2, as the mediator of TNF $\alpha$-induced increase in AMPA receptor trafficking and surface expression (He et al., 2012). Regarding the contribution of TNF $\alpha$ in response to neural injury, it has been shown that TNF $\alpha$ levels are increased in a preclinical model of spinal cord injury. In line with these results, it was reported that spinal cord injury induces trafficking of AMPA receptors to the neuronal membrane, a process that enhances excitotoxicity mediated by glutamate (Ferguson et al., 2008). Thus, the current evidence indicates that glia-derived TNF $\alpha$ evokes increased expression of AMPA receptors on post-synaptic neurons, which has been involved not only in beneficial processes such as synaptic plasticity and memory acquisition, but also in injury-triggered neurotoxicity.

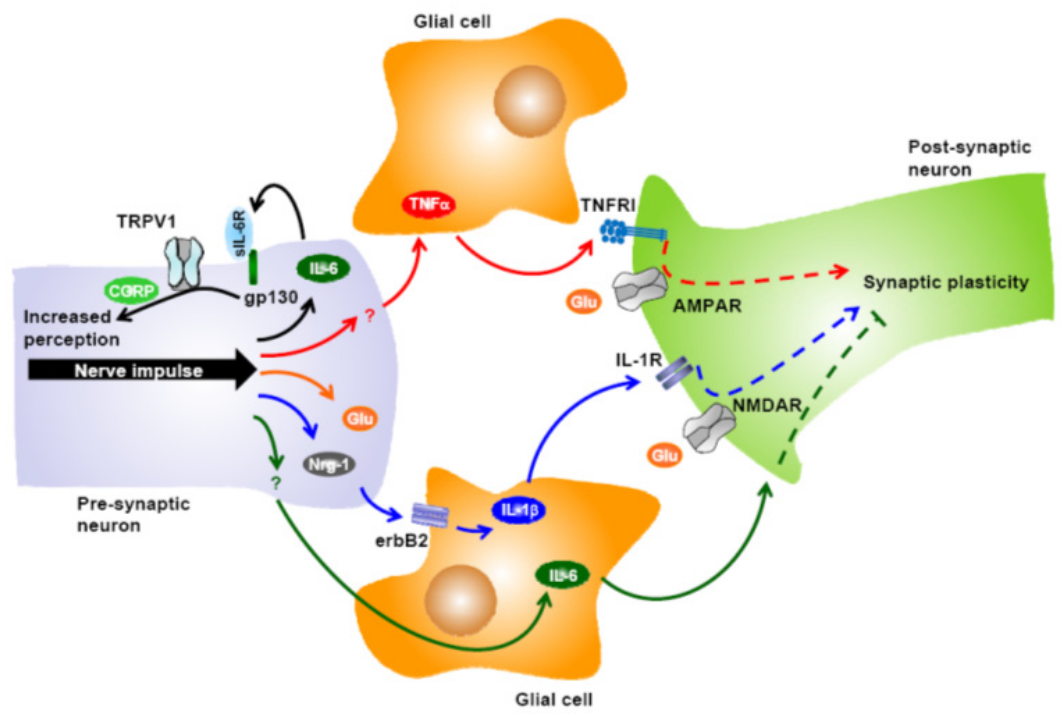

Figure 13. Cytokines as mediators between nervous system cells. Modulation of glutamate (Glu)mediated signaling in post-synaptic neurons by cytokines produced by glial cells is shown. In addition, regulation of sensitivity of afferent terminals by neuron-derived IL-6 is also shown. For explanation and abbreviations see the text. 
In contrast to the numerous studies about the role of cytokines as glia-derived mediators on nervous function, evidence showing modulation of nervous functions by neuron-derived cytokines is very limited. One of the mechanisms involving cytokine release by neurons is IL-6 secretion by primary afferent terminals into the spinal cord in neuropathic pain. It has been shown that arrival of nerve impulses induces the secretion of IL-6, along with several other neuron-derived mediators. In agreement with an autocrine loop, gp130 and gp130binding subunits of IL-6 receptor have been detected abundantly in afferent neurons under these pathological conditions, nevertheless the binding component for IL-6 itself (glycoprotein 80) has not been detected. Despite stimulation of cultured neurons with IL-6 does not induce any response, addition of a soluble fragment of its receptor results in a rapid sensitization of transient receptor potential cation channel (TRPV1) conductance in response to heat (figure 13). In addition to sensitization of TRPV1 conductance, the addition of a soluble fragment of IL-6 receptor results in the release of calcitonin gene related peptide (CGRP), a mediator involved in pain transmission. In line with a response mediated by a gp130-linked receptor, inhibitors of gp130-mediated signaling prevented the enhancement of TRPV1 sensitivity (Miller et al., 2009). Thus, taken together these results suggest an important role for neuron-derived IL-6 in chronic pain and also indicate that triggering of IL-6 signaling in neurons probably requires the production of soluble IL-6 receptors secreted from other cell types in the vicinity.

\section{Conclusion}

The interaction between immune system and nervous system involves reciprocal regulation in mammals which can be analyzed in two directions: the immune mediated regulation of nervous system function and the nervous system mediated regulation of immunity. $\mathrm{T}$ cells and dendritic cells are two pivotal players in the initiation and development of the immune response and thereby, these two kind of cells become the main convergence points through which immune system regulates nervous system and vice versa. Nervous system mediated interactions occurs not only through classical neurotransmitters produced by nervous cells and acting over immune cells, but also through cytokines derived from cells of the nervous system and exerting their effects on immune system cells. Similarly, immune system mediated regulation of nervous system function occurs by production of both cytokines and neurotransmitters by immune cells which act on nervous system cells. Nervous system mediated regulation of immunity is performed at two levels: 1, by the peripheral nervous system that innervates lymphoid organs in which it releases mediators that act subsequently over immune cells, regulating initiation and polarization of the immune response, and 2, when immune cells cross the blood brain barrier and infiltrate the central nervous system parenchyma, where their effector functions are exposed to regulation mediated by molecules produced by nervous system cells. On the other hand, immune mediated regulation of nervous system functions also occurs in two ways: 1, in the absence of inflammation, immune cells keep patrolling throughout the cerebrospinal fluid where they interact with nervous system cells regulating acquisition of memory, learning and 
behaviour, and 2, when brain damage or imbalance of glial homeostasis initiate inflammatory processes, immune cells are recruited into the central nervous system parenchyma where they produce molecules which act over glial cells and neurons. Importantly, both nervous and immune systems may produce and also respond to both kinds of mediators: neurotransmitters and cytokines. Studies performed in this area have been carried out mainly in mice, however there are several studies performed in other different mammals, including humans, cats, dogs, and other rodents, confirming results obtained with mice. Thereby, mechanisms involving neuro-immune interactions could be extrapolated to all of the mammals. Imbalance on these different kinds of neuro-immune interaction may favor the onset of autoimmune disorders, contribute to the development of tumors or susceptibility to infectious diseases and also may constitute an important component of pathogenic mechanisms involved in the neurodegenerative diseases such as Parkinson's disease and amyotrophic lateral sclerosis. Due to the similitude of these mechanisms along mammals and taking into account the vast number of murine tools currently developed, including syngenic and congenic strains and also transgenic and knockout mice, studies performed in mouse as model constitute an excellent approach to evaluate neuro-immune mechanisms in vivo.

\section{Author details}

Rodrigo Pacheco, Francisco Contreras and Carolina Prado

Fundación Ciencia y Vida,

Universidad San Sebastián and Universidad Andrés Bello,

Santiago,

Chile

\section{Acknowledgement}

We thank to supporting grants 1095114 from FONDECYT, PFB-16 from CONICYT and 2011-0001-R from Universidad San Sebastián. CP and FC hold CONICYT graduated fellowships.

\section{References}

Alaniz, R.C., Thomas, S.A., Perez-Melgosa, M., Mueller, K., Farr, A.G., Palmiter, R.D. \& Wilson, C.B. (1999). Dopamine beta-hydroxylase deficiency impairs cellular immunity. Proc Natl Acad Sci U S A, Vol. 96, No. 5, (Mar 2), pp. (2274-2278)

Amedei, A., Niccolai, E. \& D'Elios, M.M. (2011). T cells and adoptive immunotherapy: recent developments and future prospects in gastrointestinal oncology. Clin Dev Immunol, Vol. 2011, No. pp. (1-17)

Anandasabapathy, N., Victora, G.D., Meredith, M., Feder, R., Dong, B., Kluger, C., Yao, K., Dustin, M.L., Nussenzweig, M.C., Steinman, R.M. \& Liu, K. (2011). Flt3L controls 
the development of radiosensitive dendritic cells in the meninges and choroid plexus of the steady-state mouse brain. J Exp Med, Vol. 208, No. 8, (Aug 1), pp. (16951705)

Appel, S.H. (2009). CD4+ T cells mediate cytotoxicity in neurodegenerative diseases. J Clin Invest, Vol. 119, No. 1, (Jan), pp. (13-15)

Bennett, S.R., Carbone, F.R., Karamalis, F., Flavell, R.A., Miller, J.F. \& Heath, W.R. (1998). Help for cytotoxic-T-cell responses is mediated by CD40 signalling. Nature, Vol. 393, No. 6684, (Jun 4), pp. (478-480)

Besedovsky, H.O. \& del Rey, A. (2011). Central and peripheral cytokines mediate immunebrain connectivity. Neurochem Res, Vol. 36, No. 1, (Jan), pp. (1-6)

Bhowmick, S., Singh, A., Flavell, R.A., Clark, R.B., O'Rourke, J. \& Cone, R.E. (2009). The sympathetic nervous system modulates CD4(+)FoxP3(+) regulatory $\mathrm{T}$ cells via a TGFbeta-dependent mechanism. J Leukoc Biol, Vol. 86, No. 6, (Dec), pp. (1275-1283)

Cosentino, M., Fietta, A.M., Ferrari, M., Rasini, E., Bombelli, R., Carcano, E., Saporiti, F., Meloni, F., Marino, F. \& Lecchini, S. (2007). Human CD4+CD25+ regulatory T cells selectively express tyrosine hydroxylase and contain endogenous catecholamines subserving an autocrine/paracrine inhibitory functional loop. Blood, Vol. 109, No. 2, (Jan 15), pp. (632-642)

Del Prete, G. (1998). The concept of type-1 and type-2 helper T cells and their cytokines in humans. Int Rev Immunol, Vol. 16, No. 3-4, pp. (427-455)

Derecki, N.C., Cardani, A.N., Yang, C.H., Quinnies, K.M., Crihfield, A., Lynch, K.R. \& Kipnis, J. (2010). Regulation of learning and memory by meningeal immunity: a key role for IL-4. J Exp Med, Vol. 207, No. 5, (May 10), pp. (1067-1080)

Ferguson, A.R., Christensen, R.N., Gensel, J.C., Miller, B.A., Sun, F., Beattie, E.C., Bresnahan, J.C. \& Beattie, M.S. (2008). Cell death after spinal cord injury is exacerbated by rapid TNF alpha-induced trafficking of GluR2-lacking AMPARs to the plasma membrane. $J$ Neurosci, Vol. 28, No. 44, (Oct 29), pp. (11391-11400)

Franco, R., Pacheco, R., Lluis, C., Ahern, G.P. \& O'Connell, P.J. (2007). The emergence of neurotransmitters as immune modulators. Trends Immunol, Vol. 28, No. 9, (Sep), pp. (400-407)

Friedl, P., den Boer, A.T. \& Gunzer, M. (2005). Tuning immune responses: diversity and adaptation of the immunological synapse. Nat Rev Immunol, Vol. 5, No. 7, (Jul), pp. (532545)

Goshen, I., Kreisel, T., Ounallah-Saad, H., Renbaum, P., Zalzstein, Y., Ben-Hur, T., LevyLahad, E. \& Yirmiya, R. (2007). A dual role for interleukin-1 in hippocampal-dependent memory processes. Psychoneuroendocrinology, Vol. 32, No. 8-10, (Sep-Nov), pp. (11061115)

Goverman, J. (2009). Autoimmune T cell responses in the central nervous system. Nat Rev Immunol, Vol. 9, No. 6, (Jun), pp. (393-407)

Grebe, K.M., Hickman, H.D., Irvine, K.R., Takeda, K., Bennink, J.R. \& Yewdell, J.W. (2009). Sympathetic nervous system control of anti-influenza CD8+ T cell responses. Proc Natl Acad Sci U S A, Vol. 106, No. 13, (Mar 31), pp. (5300-5305) 
Harle, P., Pongratz, G., Albrecht, J., Tarner, I.H. \& Straub, R.H. (2008). An early sympathetic nervous system influence exacerbates collagen-induced arthritis via CD4+CD25+ cells. Arthritis Rheum, Vol. 58, No. 8, (Aug), pp. (2347-2355)

He, P., Liu, Q., Wu, J. \& Shen, Y. (2012). Genetic deletion of TNF receptor suppresses excitatory synaptic transmission via reducing AMPA receptor synaptic localization in cortical neurons. Faseb J, Vol. 26, No. 1, (Jan), pp. (334-345)

Kawashima, K. \& Fujii, T. (2003). The lymphocytic cholinergic system and its contribution to the regulation of immune activity. Life Sci, Vol. 74, No. 6, (Dec 26), pp. (675-696)

Kawashima, K., Yoshikawa, K., Fujii, Y.X., Moriwaki, Y. \& Misawa, H. (2007). Expression and function of genes encoding cholinergic components in murine immune cells. Life Sci, Vol. 80, No. 24-25, (May 30), pp. (2314-2319)

Kipnis, J., Cardon, M., Avidan, H., Lewitus, G.M., Mordechay, S., Rolls, A., Shani, Y. \& Schwartz, M. (2004). Dopamine, through the extracellular signal-regulated kinase pathway, downregulates CD4+CD25+ regulatory T-cell activity: implications for neurodegeneration. J Neurosci, Vol. 24, No. 27, (Jul 7), pp. (6133-6143)

Lanzavecchia, A. \& Sallusto, F. (2001). Regulation of T cell immunity by dendritic cells. Cell, Vol. 106, No. 3, (Aug 10), pp. (263-266)

Lucin, K.M. \& Wyss-Coray, T. (2009). Immune activation in brain aging and neurodegeneration: too much or too little? Neuron, Vol. 64, No. 1, (Oct 15), pp. (110122)

McGeachy, M.J. \& Cua, D.J. (2008). Th17 cell differentiation: the long and winding road. Immunity, Vol. 28, No. 4, (Apr), pp. (445-453)

Mignini, F., Streccioni, V. \& Amenta, F. (2003). Autonomic innervation of immune organs and neuroimmune modulation. Auton Autacoid Pharmacol, Vol. 23, No. 1, (Feb), pp. $(1-25)$

Miller, R.J., Jung, H., Bhangoo, S.K. \& White, F.A. (2009). Cytokine and chemokine regulation of sensory neuron function. Handb Exp Pharmacol, Vol. No. 194, pp. (417449)

Miura, T., Kudo, T., Matsuki, A., Sekikawa, K., Tagawa, Y., Iwakura, Y. \& Nakane, A. (2001). Effect of 6-hydroxydopamine on host resistance against Listeria monocytogenes infection. Infect Immun, Vol. 69, No. 12, (Dec), pp. (7234-7241)

Nouri-Shirazi, M., Banchereau, J., Fay, J. \& Palucka, K. (2000). Dendritic cell based tumor vaccines. Immunol Lett, Vol. 74, No. 1, (Sep 15), pp. (5-10)

Pacheco, R., Prado, C.E., Barrientos, M.J. \& Bernales, S. (2009). Role of dopamine in the physiology of T-cells and dendritic cells. J Neuroimmunol, Vol. 216, No. 1-2, (Nov 30), pp. (8-19)

Pacheco, R., Riquelme, E. \& Kalergis, A.M. (2010). Emerging evidence for the role of neurotransmitters in the modulation of $\mathrm{T}$ cell responses to cognate ligands. Cent Nerv Syst Agents Med Chem, Vol. 10, No. 1, (Mar), pp. (65-83)

Park, T.S., Rosenberg, S.A. \& Morgan, R.A. (2011). Treating cancer with genetically engineered T cells. Trends Biotechnol, Vol. 29, No. 11, (Nov), pp. (550-557) 
Parker, D.C. (1993). T cell-dependent B cell activation. Annu Rev Immunol, Vol. 11, No. pp. (331-360)

Prado, C., Contreras, F., Gonzalez, H., Diaz, P., Elgueta, D., Barrientos, M., Herrada, A.A., Lladser, A., Bernales, S. \& Pacheco, R. (2012). Stimulation of dopamine receptor d5 expressed on dendritic cells potentiates th17-mediated immunity. J Immunol, Vol. 188, No. 7, (Apr 1), pp. (3062-3070)

Ren, K. \& Dubner, R. (2010). Interactions between the immune and nervous systems in pain. Nat Med, Vol. 16, No. 11, (Nov), pp. (1267-1276)

Reynolds, A.D., Stone, D.K., Hutter, J.A., Benner, E.J., Mosley, R.L. \& Gendelman, H.E. (2010). Regulatory $\mathrm{T}$ cells attenuate th17 cell-mediated nigrostriatal dopaminergic neurodegeneration in a model of Parkinson's disease. J Immunol, Vol. 184, No. 5, (Mar 1), pp. (2261-2271)

Ridge, J.P., Di Rosa, F. \& Matzinger, P. (1998). A conditioned dendritic cell can be a temporal bridge between a CD4+ T-helper and a T-killer cell. Nature, Vol. 393, No. 6684, (Jun 4), pp. (474-478)

Rosas-Ballina, M., Olofsson, P.S., Ochani, M., Valdes-Ferrer, S.I., Levine, Y.A., Reardon, C., Tusche, M.W., Pavlov, V.A., Andersson, U., Chavan, S., Mak, T.W. \& Tracey, K.J. (2011). Acetylcholine-synthesizing $\mathrm{T}$ cells relay neural signals in a vagus nerve circuit. Science, Vol. 334, No. 6052, (Oct 7), pp. (98-101)

Rosas-Ballina, M. \& Tracey, K.J. (2009). The neurology of the immune system: neural reflexes regulate immunity. Neuron, Vol. 64, No. 1, (Oct 15), pp. (28-32)

Schoenberger, S.P., Toes, R.E., van der Voort, E.I., Offringa, R. \& Melief, C.J. (1998). T-cell help for cytotoxic T lymphocytes is mediated by CD40-CD40L interactions. Nature, Vol. 393, No. 6684, (Jun 4), pp. (480-483)

Schoenborn, J.R. \& Wilson, C.B. (2007). Regulation of interferon-gamma during innate and adaptive immune responses. Adv Immunol, Vol. 96, No. pp. (41-101)

Smith, K.M., Pottage, L., Thomas, E.R., Leishman, A.J., Doig, T.N., Xu, D., Liew, F.Y. \& Garside, P. (2000). Th1 and Th2 CD4+ T cells provide help for B cell clonal expansion and antibody synthesis in a similar manner in vivo. J Immunol, Vol. 165, No. 6, (Sep 15), pp. (3136-3144)

Stellwagen, D. \& Malenka, R.C. (2006). Synaptic scaling mediated by glial TNF-alpha. Nature, Vol. 440, No. 7087, (Apr 20), pp. (1054-1059)

Watford, W.T., Moriguchi, M., Morinobu, A. \& O'Shea, J.J. (2003). The biology of IL-12: coordinating innate and adaptive immune responses. Cytokine Growth Factor Rev, Vol. 14, No. 5, (Oct), pp. (361-368)

Wong, P. \& Pamer, E.G. (2003). CD8 T cell responses to infectious pathogens. Annu Rev Immunol, Vol. 21, No. pp. (29-70)

Yirmiya, R. \& Goshen, I. (2011). Immune modulation of learning, memory, neural plasticity and neurogenesis. Brain Behav Immun, Vol. 25, No. 2, (Feb), pp. (181-213) 
Zheng, Y., Manzotti, C.N., Liu, M., Burke, F., Mead, K.I. \& Sansom, D.M. (2004). CD86 and CD80 differentially modulate the suppressive function of human regulatory T cells. $J$ Immunol, Vol. 172, No. 5, (Mar 1), pp. (2778-2784) 